Richard Stang, Christine Gläser, Hans-Dieter Weckmann und Fabian Franke

\title{
Zur Situation der Lernwelt Hochschule
}

\author{
Grundlagen des Projektes Lernwelt Hochschule
}

\section{Einleitung}

Die Veränderungen im Rahmen des Bologna-Prozesses hatten und haben gravierende Auswirkungen auf die Hochschulen. Dabei haben sich Herausforderungen für Hochschulen auf den unterschiedlichsten Ebenen ergeben. Sursock hatte 2015 auf der Basis einer Befragung von 451 Hochschulen folgende Trends konstatiert: stärkere Internationalisierung, größere Bedeutung von Informations- und Kommunikationstechniken, größere Autonomie der Hochschulen, stärkere Vernetzung, heterogenere Studierendenstruktur, veränderte Finanzierungsstrukturen, veränderte Studierenden- und Lehrendenrollen, zunehmende Bedeutung neuer Lehrmethoden und Veränderungen bei den Lerninfrastrukturen, unter anderem durch Etablierung von Lernzentren (Sursock 2015, 22-94).

Der NMC-Horizon-Report 2018 zeigt auf, welche neuen Herausforderungen der technologische Wandel für die Hochschulen mit sich bringt und weist auf sechs Schlüsseltrends bei Bildungstechnologien hin, die für Hochschulen Auswirkungen haben werden (Adams Becker et al. 2018, 8-21):

- kurzfristige Auswirkungen (ein bis zwei Jahre): Growing Focus on Measuring Learning, Redesigning Learning Spaces;

- mittelfristige Auswirkungen (drei bis fünf Jahre): Proliferation of Open Educational Resources; The Rise of New Forms of Interdisciplinary Studies;

- langfristige Auswirkungen (fünf oder mehr Jahre): Advancing Cultures of Innovation, Cross-Institution \& Cross-Sector Collaboration.

Dabei ergeben sich folgende zentralen Herausforderungen (Adams Becker et al. 2018, 22-35):

- Lösbare Herausforderungen: Authentic Learning Experiences, Improving Digital Literacy;

- Schwierig zu lösende Herausforderungen: Adapting Organizational Designs to the Future of Work, Advancing Digital Equity;

- Sehr schwer zu lösende Herausforderungen: Economic and Political Pressures, Rethinking the Roles of Educators. 
Als konkrete Entwicklungslinien werden folgende prognostiziert (Adams Becker et al. 2018, 36-49):

- mit einem Zeithorizont von bis zu einem Jahr: Analytics Technologies, Makerspaces;

- mit einem Zeithorizont von zwei bis drei Jahren: Adaptive Learning Technologies, Artificial Intelligence;

- mit einem Zeithorizont von vier bis fünf Jahren: Mixed Reality, Robotics.

Betrachtet man nun die von Sursock (2015) und Adams Becker et al. (2018) herausgearbeiteten Trends, wird deutlich, dass Hochschulen vor immensen Herausforderungen stehen. Dies gilt nicht nur bezogen auf das Thema Digitalisierung und dem damit verbundenen Einsatz von Informations- und Kommunikationstechniken, sondern auch bezogen auf die traditionellen Elemente von Bildung, wie die Gestaltung didaktischer Konzepte in Form von veränderten Lehr- und Lernformen, die Veränderung der Rolle der Studierenden und der Lehrenden sowie die Neukonzeptionierung von Lehr- und Lernräumen.

\section{Shift from Teaching to Learning}

Bereits Mitte der 1990er Jahre wurde in den USA intensiv über die Frage diskutiert, welches Lehr-Lern-Paradigma die Hochschulen in Zukunft leiten soll. Barr und Tagg beschrieben den notwendigen Paradigmenwechsel wie folgt:

A paradigm shift is taking hold in American higher education. In the briefest form, the paradigm that has governed our colleges is this: A college is an institution that exists to provide instruction. Subtly but profoundly we are shifting to a new paradigm: A college is an institution that exists to produce learning. This shift changes everything. It is both needed and wanted. (Barr/Tagg 1995, 12, H. i. O.)

Im Zuge der Bologna-Reform gilt die Kompetenzorientierung, die auch den Shift from Teaching to Learning umfasst, als grundlegendes Konzept für die Hochschulen. Hierbei ist die Orientierung weg von der Inhalts- und Wissensvermittlung hin $\mathrm{zu}$ den Lernergebnissen der Studierenden leitend, um diese auf die komplexe, sich ständig verändernde Arbeits- und Beschäftigungswelt vorzubereiten. Dem Anwendungs- und Praxisbezug kommt dabei eine große Bedeutung zu.

Um diese Kompetenzorientierung konsequent zu unterstützen, müssen die Hochschulen Veränderungen auf allen Ebenen - nicht nur im engeren Bereich der Lehre - gestalten. Es sind an den Hochschulen für die Kompetenzorientie- 
rung Umsetzungsszenarien zu entwickeln (Weyer et al. 2017, 9). Dieser Wechsel vom Instruktions-Paradigma zum Lern-Paradigma wird auch in Deutschland immer wieder als Herausforderung formuliert, doch ist er bislang nur unzureichend in eine pädagogische Strategieentwicklung für Hochschulen einbezogen worden (Brahm et al. 2016).

Ein solcher Paradigmenwechsel ist mit veränderten didaktischen Konzepten wie zum Beispiel dem Projektlernen verbunden. Auf der Umsetzungsebene erfordert eine solche Ausrichtung eine Supportstruktur, die sich an den Bedarfen von Einzellernenden und Lern- bzw. Projektgruppen orientiert. In diesem Kontext spielen die Hochschulbibliotheken eine zentrale Rolle, da sie seminarund vorlesungsunabhängige Lern- und Raumangebote bereithalten (Stang 2016, 72-97).

Bibliotheken entwickeln sich immer stärker zu Lernzentren innerhalb der Hochschulen. In den letzten beiden Jahrzehnten sind vor allem die internationalen Entwicklungen zu Learning Commons und Learning Centers Inspiration und Treiber der Entwicklungen in Deutschland. Diese Konzepte weisen typische Strukturelemente auf, wie eine Vielfalt an Arbeitsplätzen und Zonierungen, die vor allem das Arbeiten in Gruppen unterstützen. Die Ausstattung (Mobiliar und Geräte) ist flexibel und anpassbar und die IT-Infrastruktur auf aktuellem technischen Stand. Ergänzt werden die Konzepte durch Services zu inhaltlichen und technischen Fragen.

Oft ausgehend von der Initiative von Bibliotheken werden kooperative Konzepte immer stärker ausgebaut - über Bibliotheks-, IT- und Mediensupport hinaus zu Studierendenservices, Studienberatung, Schreibzentren -, um den Studierenden alle lernrelevanten Dienstleistungen unter einem Dach anbieten zu können. Auf diese Weise entstehen bottom-up zunehmend hochschulweite Konzepte der Lernwelt Hochschule.

Auch die Gestaltung von E-Learning-Angeboten und -Umgebungen ist eine große Herausforderung für die Hochschulen (Reiners 2017). Um digitale Medien optimal in die Hochschule einzubinden, bedarf es flexibler Raumstrukturen. So vervielfältigen sich lernrelevante Orte (Seitter 2001, 225.), der Bedarf an Lernräumen wächst sowohl quantitativ als auch qualitativ. Die klassischen und formalen Lehr- und Lernszenarien in Hochschulen werden dadurch zunehmend entgrenzt, und die Verbindung von formalen, non-formalen und informellen Lernumgebungen wird immer wichtiger. Lehren und Lernen rücken in der Umsetzung immer näher zusammen. Dies bedeutet auch, dass dies Niederschlag in den Konzeptelementen für Lehr- und Lernräume finden muss (Stang 2017). Räume der formalen Lehre wandeln sich auch zunehmend; die Bedarfe nach mehr Interaktion, Medieneinsatz und Flexibilität finden Eingang in die Raumkonzepte. 
An diesen Entwicklungen wird der Bedarf nach einer ganzheitlichen Sicht und strategischen Perspektive auf die Lernraumentwicklungen deutlich. Die Perspektive der Lernenden auf die Lernraumsituation ist weniger geprägt von Strukturen als durch Lern- und Organisationsprozesse. Diese sollen im Rahmen ihrer Aktivitäten so komfortabel wie möglich verbunden werden. Studierende sind darauf angewiesen, dass sie flexibel und ohne Brüche agieren können, um verschiedene Lernaktivitäten im Team oder allein, an verschiedenen Orten, mit wechselnden Infrastrukturen und Räumen sowie unter Nutzung bedarfsgerechter Services, wie Informationsdiensten oder technischem Support, umsetzen zu können.

Der Gestaltung von physischen, digitalen und hybriden Lernräumen kommt in diesem Zusammenhang in Zukunft eine wichtige Funktion zu. Allerdings müssen damit auch veränderte Organisationskonzepte verbunden sein, die die Hochschule als ganzheitliche sowie integrierte Lernwelt etablieren und nicht als segmentierte und additive, wie es derzeit in den meisten Hochschulen der Fall ist.

Lernräume an Hochschulen werden bisher zumeist unter baulich sichtbaren und digitalen Aspekten betrachtet (Rummler 2014). Doch sinnvolle Lernraumgestaltung erfordert mehr als nur (innen-)architektonische und informationstechnische Betrachtungen. Es geht vielmehr um das Zusammenspiel von Raumstruktur, technischer Infrastruktur, didaktischen Konzepten und organisatorischer Rahmung. Die Lernwelt Hochschule ist mehr als nur „Räume zum Lernen“.

Die Hochschule als multidimensionale Organisation muss heute komplexen Anforderungen mit vielfältigen Lernsettings und Serviceleistungen begegnen. Dies erfordert ein Zusammenspiel von strategischer Hochschulentwicklung und den verschiedenen Bereichen der Hochschulinfrastruktur, wie zum Beispiel der Bibliothek, dem Didaktikzentrum, der IT-Abteilung bzw. dem Rechenzentrum.

Erste Ansätze hierzu werden im Rahmen der Deutschen Initiative für Netzwerkinformation e. V. (DINI) umgesetzt, in der sich Wissenschaftliche Bibliotheken, Medienzentren, Rechenzentren sowie weitere Wissenschaftseinrichtungen und -organisationen zusammengeschlossen haben. Seit vielen Jahren zeigt DINI Handlungsfelder und Wege auf, um die Serviceleistungen für Forschung und Lehre mit innovativen Informations- und Kommunikationstechnologien effizient zu gestalten (DINI 2018). In ihren Empfehlungen „Die Hochschule zum Lernraum entwickeln“ (DINI 2013) hat sie bereits 2013 die Vorteile einer hochschulweiten Strategie aufgezeigt und die „Entgrenzung von Zuständigkeiten“ gefordert. Die Empfehlungen betonen die Notwendigkeit der Vielfalt physischer und digitaler Lernräume und enthalten zahlreiche Praxisbeispiele, wie im Zusammenspiel verschiedener Einrichtungen an Hochschulen studentische Arbeits- 
plätze, Kursräume und virtuelle Lernräume geschaffen und hochschulweite Strategien zur Förderung von Informationskompetenz umgesetzt werden können.

Einzelne Good Practice-Beispiele können jedoch nicht darüber hinwegtäuschen, dass dieses Zusammenspiel nur selten sehr weit entwickelt ist. So agieren einzelne Einrichtungen der Hochschule oftmals von der Gesamtstrategie der Hochschule - wenn es diese gibt - und untereinander unabhängig. Dabei können Parallelstrukturen entstehen, die nicht nur für die Studierenden undurchsichtig sind. Eine studierendenorientierte Hochschule, die den Paradigmenwechsel vom Lehren zum Lernen vollziehen will, sollte aber für durchsichtige und nachvollziehbare Strukturen sorgen. Dies bedeutet, dass die Prozesse der Studierenden bei der Entwicklung der Hochschule im Fokus stehen sollten und weniger die traditionellen Organisationsstrukturen der Hochschulen beziehungsweise derer Einrichtungen. Es erfordert eine Gesamtstrategie bezogen auf die Gestaltung der Lernwelt Hochschule, um die Studierenden optimal bei der Bewältigung ihrer Aufgaben zu unterstützen.

\section{Von der Analyse zum Projekt}

Veränderungsprozesse strategisch anzugehen, bedarf eines fundierten und strukturierten Überblicks über die aktuelle Situation der Hochschullandschaft in Deutschland. Dabei geht es vor allem auch um einen Überblick über mögliche Problemstellungen und denkbarer Lösungsansätze für die sinnvolle, ganzheitliche Lernweltgestaltung und der dafür notwenigen Strukturen und Prozesse. Im Rahmen der Kooperation des Learning Research Centers der Hochschule der Medien Stuttgart und der AG Lernräume der Deutschen Initiative für Netzwerkinformation e.V. (DINI) gab es bereits 2014 Überlegungen, eine Handreichung für Hochschulen zur Gestaltung von Lernwelten zu entwickeln. Bei der Recherche nach Analysen der Hochschulsituation unter der oben beschriebenen ganzheitlichen Perspektive, zeigte sich allerdings, dass solche Daten wenn überhaupt - nur in Teilbereichen vorlagen. Das war der Ausgangspunkt für die Überlegungen, dieses Defizit zu beheben und im Rahmen eines Projektes zu bearbeiten.

Durch Förderung der Dieter Schwarz Stiftung konnte im Januar 2017 das Forschungsprojekt Lernwelt Hochschule gestartet werden. Das Projekt wurde in Kooperation von der Hochschule der Medien Stuttgart (Projektleitung), der DINI, der Otto-Friedrich-Universität Bamberg, der Heinrich-Heine-Universität 
Düsseldorf und der Hochschule für angewandte Wissenschaften Hamburg bearbeitet.

Um einen strukturierten Überblick über die Ist-Situation der Hochschulen in Deutschland zu gewinnen, wurden im Rahmen einer umfangreichen OnlineBefragung alle deutschen Hochschulen angeschrieben. Unter Hochschulen wurden im Projekt Universitäten und gleichgestellte Hochschulen (Technische Hochschulen, Technische Universitäten, Pädagogische Hochschulen, Theologische Hochschulen u. a.), Kunst- und Musikhochschulen, Fachhochschulen (Hochschulen für angewandte Wissenschaften, Hochschulen für angewandte Forschung) sowie sonstige staatliche oder staatlich anerkannte Hochschulen, wie zum Beispiel private Hochschulen, Berufsakademien oder Duale Hochschulen, subsummiert.

Unter der Perspektive, ein kooperatives, integratives Konzept zur Gestaltung der Lernwelt Hochschule im (infra-)strukturellen Bereich $\mathrm{zu}$ entwickeln, wie es zum Beispiel bereits in Großbritannien vorliegt (UCISA 2016), ging es vor allem darum, neben der Ausstattung auch die studierendenbezogenen Dienstleistungen, die für das erfolgreiche Lernen von Relevanz sind, in den Fokus zu rücken. Inhaltliche Schwerpunkte des Projektes waren ein Blick auf die vier Bereiche Hochschulorganisation, Hochschuldidaktik, physische Lehr- und Lernräume und digitale Strukturen. An diesen Bereichen orientierten sich auch die Untersuchungsdimensionen der Studie, die folgende Aspekte in den Fokus rückten:

- Hochschulorganisation: Hierzu gehören die Strategie der Hochschule, aber auch die Organisationsstruktur. Die Angebote und Services für Studierende werden untersucht. Interessant sind dabei Aspekte der Koordination und Zusammenarbeit unterschiedlicher Abteilungen und Einrichtungen der Hochschule.

- Hochschuldidaktik: Hier soll geklärt werden, wie weit der Shift from Teaching to Learning in den Hochschulen bereits thematisiert und umgesetzt wird. Im Bereich der Hochschuldidaktik wird nach konkreten Angeboten für Lehrende gefragt. Es wird untersucht, inwieweit die Ausrichtung der Lehre auf Kompetenzorientierung erfolgt.

- Digitale Strukturen: Zur Klärung des Ausbaustandes der digitalen Infrastrukturen und Prozesse werden Basisdienste wie Internetanbindung und Systeme wie Verwaltungssoftware oder Lernplattformen abgefragt. Ein besonderes Interesse gilt den Social Media-Tools der Studierenden und der Frage, wie diese in die digitale Hochschule eingebunden werden können.

- Physische Lehr- und Lernräume: Ziel ist es, einen Überblick der Standorte und Räume in den Hochschulen zu erhalten. Informationen zum Raummanagement und den Verantwortlichkeiten sind ebenso relevant wie die Nutzungsstruktur der Räumlichkeiten. Es wird nach konkreten Ausstattungen 
und Öffnungszeiten gefragt. Dabei spielen Selbstlernareale eine besondere Rolle.

Dazu wurden bei der Online-Befragung in den Hochschulen (Becker/Stang 2020) jeweils vier Zielgruppen angesprochen: Hochschulleitungen sowie Bibliotheksleitungen, Leitungen der IT-Abteilungen und Leitungen der Infrastruktur-Abteilungen.

Bei der Vorbereitung der Online-Befragung zeigte sich, dass in vielen der in den Fokus gerückten Bereiche keine strukturierten Daten vorlagen. So gibt es bezogen auf die räumliche Infrastruktur keine einheitliche Übersicht für die deutschen Hochschulen, und auch die meisten Hochschulen verfügen über keine differenzierte Übersicht, die zum Beispiel auch Selbstlernarbeitsplätze einbezieht. Dies zeigte sich bei der Anfrage an Bauämter, die für die Hochschulen zuständig waren.

Aufgrund der ersten Erkenntnisse der Auswertung der Online-Befragung zeigte sich die Notwendigkeit, die Dokumentenanalyse zu intensivieren. Hierbei wurden vor allem die Landeshochschulgesetze, Zielvereinbarungen der Hochschulen mit dem jeweiligen Bundesland, die Entwicklungs- und Strukturpläne sowie Leitbilder der jeweiligen Hochschulen - soweit die Dokumente öffentlich verfügbar waren - genauer im Hinblick auf Aussagen bezüglich der Gestaltung der Lernwelt Hochschule untersucht (Weichert 2020a; Weichert 2020b).

Auf der Basis der Auswertung der Ergebnisse der Online-Befragung wurden Expertinnen und Experten aus den Zielgruppen Hochschulleitungen sowie Bibliotheksleitungen, Leitungen der IT-Abteilungen und Leitungen der Infrastruktur-Abteilungen mit Hilfe von Leitfadeninterviews befragt (Aschinger 2020). Die Interviewteilnehmenden wurden so ausgewählt, dass sie die im Rahmen der Online-Befragung als Good Practice für die einzelnen Bereiche eruierten Akteurinnen und Akteure repräsentierten.

Um die Perspektive der Studierenden einzubeziehen, wurden ergänzend zur Online-Befragung und den Leitfadeninterviews mit Expertinnen und Experten Fallstudien an ausgewählten Good Practice-Hochschulen durchgeführt (Gläser/Kobsch 2020). Dabei kamen ethnografische Methoden zum Einsatz, um den Perspektivwechsel zu unterstützen. Den Fallstudien kamen im Rahmen des Projekts zwei wesentliche Aufgaben zu: Zum einen ging es um die Illustration und Erfassung der studentischen Perspektive. Mit Hilfe der Fallstudien sollte gezeigt werden, welche typischen Abläufe aus Studierendensicht gut funktionieren und in welchen Bereichen zum Beispiel aufgrund der Verwaltungsprozesse Probleme für die Studierenden entstehen. Es sollte eine deutliche Vorstellung entwickelt werden, wie Studierende ihre Aufgaben bewältigen und wie sie die Lernwelt Hochschule erleben. Dabei sollte sich die Forschung nicht auf die 
Lernprozesse beschränken, sondern auch Einsichten in den Kontext der Lernaktivitäten von Studierenden ermöglichen. Zum anderen sollte durch Campusbegehungen und -beobachtungen das Umfeld der Studierenden genauer in den Blick genommen werden.

Im Projekt sollte allerdings nicht nur ein Blick auf die deutsche Situation gerichtet werden. Mit dem Einbeziehen internationaler Perspektiven (Weichert/ Stang 2020) kann deutlich gemacht werden, welche Dynamik sich derzeit weltweit - allerdings vor allem auch in Europa - bei der Gestaltung des Shift from Teaching to Learning und der Lernwelt Hochschule entwickelt.

Ein Ziel des Projektes war neben der wissenschaftlichen Aufbereitung des aktuellen Stands der Dinge, auf der Basis der Ergebnisse eine Plattform aufzubauen, auf der sich Akteurinnen und Akteure sowie Entscheiderinnen und Entscheider über Eckdaten und die Praxis der Gestaltung der Lernwelt Hochschule in den einzelnen Hochschulen informieren können. Diese Plattform soll auch als Austausch- und Informationsangebot für die Scientific Community zur Verfügung gestellt werden. Dabei ist unter wissenschaftlicher Perspektive ein weiteres Ziel, ein nachhaltiges Erhebungsverfahren zur Lernwelt Hochschule zu entwickeln, um eine belastbare Grundlage für weiterführende Studien zu schaffen.

\section{Perspektiven}

Ein zentrales Anliegen des Projektes Lernwelt Hochschule war, einen Überblick über den State-of-the-Art bei der Entwicklung eines studierendenorientierten Zugangs bei der Strategieentwicklung von Hochschulen in Deutschland zu erhalten. Bei der wissenschaftlichen Bearbeitung wurde deutlich, dass es in vielen Bereichen an einer fundierten, strukturierten Datenbasis fehlt. Das hier ein erhöhter Bedarf an Informationen von Seiten der Hochschulpraxis vorhanden ist, zeigte sich auch auf der Konferenz Zukunft Lernwelt Hochschule, die mit den Zielgruppen Hochschulleitungen, Bibliotheksleitungen, Leitungen von IT-Abteilungen und Leitungen von Infrastruktur-Abteilungen im Rahmen des Projektes durchgeführt wurde (Stang/Becker 2020).

Das Projekt konnte mit seinen Ergebnissen eine erste Basis schaffen, einen Überblick über die Lernwelt Hochschule in Deutschland zu erhalten - nicht mehr. Die derzeitigen Diskussionen und die Suchbewegungen in der deutschen Hochschullandschaft, die Bewältigung gesellschaftlicher Herausforderungen nicht nur zu begleiten, sondern auch mitzugestalten, gibt Hoffnung, dass hier etwas in Bewegung gekommen ist, was zu veränderten Strategien der Hoch- 
schulentwicklung führen wird. Dabei werden unter anderen für Veränderungsprozesse von großer Relevanz sein:

- die Integration veränderter pädagogischer Konzeptionen als Basis von Lehre,

- die Entwicklung veränderter Raumkonzepte, die Lehr- und Lernprozesse flexibel gestalten lassen,

- die Gestaltung der Hochschulorganisation als agiles und atmendes System,

- die Auflösung von hierarchischen Strukturen, die eine flexible Anpassung an sich immer schneller verändernde Rahmenbedingungen erst möglich machen wird,

- die stärkere Vernetzung der Hochschulakteurinnen und -akteure, wenn es um die Entwicklung und Umsetzung einer für das jeweilige Umfeld angepassten Strategie geht,

- die Intensivierung der Außenbeziehungen zu Partnerinnen und Partnern aus der Kommune, der Wirtschaft und sonstigen potenziellen Stakeholdern.

Viele Hochschulen haben bereits damit begonnen, ihre Strategie den zukünftigen Herausforderungen anzupassen. Die rechtlichen und politischen Rahmenbedingungen in Deutschland sowie die Struktur der finanziellen Rahmenbedingungen für Innovationsprozesse behindern derzeit noch eine stärkere Dynamik der Prozesse. Es bleibt zu hoffen, dass durch die internationalen Entwicklungen, vor deren Hintergrund die deutsche Hochschullandschaft tendenziell zunehmend ins Hintertreffen gerät, die Notwendigkeit gravierender Veränderungen erkannt wird. Zumindest erste Anzeichen lassen sich feststellen.

\section{Literatur}

Adams Becker, S.; Brown, M.; Dahlstrom, E.; Davis, A.; DePaul, K.; Diaz, V.; Pomerantz, J. (2018): NMC Horizon Report. 2018 Higher Education Edition. Louisville, CO: EDUCAUSE. https://library.educause.edu/resources/2018/8/2018-nmc-horizon-report.

Aschinger, F.; Becker, A.; Gageur, N.; Weichert, H. (2020): Forschungsfeld Lernwelt Hochschule. Methodische Zugänge zur Analyse einer differenzierten Struktur. In: A. Becker; R. Stang (Hrsg.): Lernwelt Hochschule. Dimensionen eines Bildungsbereichs im Umbruch. Berlin; Boston: De Gruyter Saur, 20-46.

Barr, R. B.; Tagg, J. (1995): From Teaching to Learning. A New Paradigm for Undergraduate Education. Change: The Magazine of Higher Learning 27/6, 12-26, DOI: 10.1080/ 00091383.1995.10544672.

Becker, A.; Stang, R. (2020): Lernwelt Hochschule im Aufbruch. Zentrale Ergebnisse einer Befragung. In: A. Becker; R. Stang (Hrsg.): Lernwelt Hochschule. Dimensionen eines Bildungsbereichs im Umbruch. Berlin; Boston: De Gruyter Saur, 71-123. 
Brahm, T.; Jenert, T.; Euler, D. (2016): Pädagogische Hochschulentwicklung als Motor für die Qualitätsentwicklung von Studium und Lehre. In: T. Brahm; T. Jenert; D. Euler (Hrsg.): Pädagogische Hochschulentwicklung. Von der Programmatik zur Implementierung. Wiesbaden: Springer VS, 19-36.

DINI - Deutsche Initiative für Netzwerkinformation. e. V. (2013): Die Hochschule zum Lernraum entwickeln. Empfehlungen der DINI-AG „Lernräume“. http://nbn-resolving.de/urn:nbn: de:0002-36551.

DINI - Deutsche Initiative für Netzwerkinformation e. V. (2018): Thesen zur Informations- und Kommunikationsinfrastruktur der Zukunft. https://edoc.hu-berlin.de/handle/18452/ 19876.

Gläser, C.; Kobsch, L. (2020): Student Experience in der Lernwelt Hochschule. Studierende im Fokus der Fallstudien. In: A. Becker; R. Stang (Hrsg.): Lernwelt Hochschule. Dimensionen eines Bildungsbereichs im Umbruch. Berlin; Boston: De Gruyter Saur, 151-170.

Reiners, A. (2017): Mobiles Lernen an Hochschulen. Formen der Umsetzung. In: F. Thissen (Hrsg.): Lernen in virtuellen Räumen. Perspektiven des Mobilen Lernens. Berlin; Boston: De Gruyter Saur, 73-87.

Rummler, K. (Hrsg.) (2014): Lernräume gestalten. Bildungskontexte vielfältig denken. Münster; New York: Waxmann.

Seitter, W. (2001): Zwischen Proliferation und Klassifikation. Zeitschrift für Erziehungswissenschaft 4/2, 225-238.

Stang, R. (2016): Lernwelten im Wandel. Entwicklungen und Anforderungen bei der Gestaltung zukünftiger Lernumgebungen. Berlin; Boston: De Gruyter Saur.

Stang, R. (2017): Lernraumgestaltung an Universitäten. Zur Relevanz physischer Lernräume im Kontext der Digitalisierung. Erziehungswissenschaft, 28/55, 29-36.

Stang, R.; Becker, A. (Hrsg.) (2020): Zukunft Lernwelt Hochschule. Perspektiven und Optionen für eine Neuausrichtung. Berlin; Boston: De Gruyter Saur.

Sursock, A. (2015): Trends 2015: Learning and Teaching in European Universities. Brüssel: European University Association. http://eua.be/Libraries/publications-homepage-list/ EUA_Trends_2015_web.pdf?sfvrsn=18.

UCISA - Universities and Colleges Information System (2016): The UK Higher Education Learning Space Toolkit. http://www.ucisa.ac.uk/learningspace.

Weichert, H. (2020a): Dimensionen gesetzlicher Rahmungen. Perspektiven deutscher Hochschulgesetzgebung. In: A. Becker; R. Stang (Hrsg.): Lernwelt Hochschule. Dimensionen eines Bildungsbereichs im Umbruch. Berlin; Boston: De Gruyter Saur, 47-58.

Weichert, H. (2020b): Strukturentwicklungspläne und Leitbilder. Orientierungen für strategische Planungen. In: A. Becker; R. Stang (Hrsg.): Lernwelt Hochschule. Dimensionen eines Bildungsbereichs im Umbruch. Berlin; Boston: De Gruyter Saur, 59-70.

Weichert, H.; Stang, R. (2020): Der Blick von außen. Einschätzungen internationaler Expertinnen und Experten. In: A. Becker; R. Stang (Hrsg.): Lernwelt Hochschule. Dimensionen eines Bildungsbereichs im Umbruch. Berlin; Boston: De Gruyter Saur, 171-182.

Weyer, E.; Wachendorf, N. M.; Mörth, A. (2017): Kompetenzorientierung, wie ist das gemeint? In: E. Cendon; N. Donner; U. Elsholz; A. Jandrich; A. Mörth; N. M. Wachendorf; E. Weyer (Hrsg.) Die kompetenzorientierte Hochschule. Kompetenzorientierung als MainstreamingAnsatz in der Hochschule. Berlin: BMBF, 6-12. https://www.pedocs.de/volltexte/2017/ 14563/pdf/Cendon_etal_2017_Die_kompetenzorientierte_Hochschule.pdf. 